\title{
Treatment of a large osseous defect in a feline tarsus using a stem cell-seeded custom implant.
}

\author{
Noel Fitzpatrick \\ Fitzpatrick Referrals, Eashing, Godalming, Surrey, United Kingdom. GU7 2QQ. \\ Cameron Black \\ Fitzpatrick Referrals, Eashing, Godalming, Surrey, United Kingdom. GU7 2QQ. \\ Melissa Choucourn \\ Fitzpatrick Referrals, Eashing, Godalming, Surrey, United Kingdom. GU7 2QQ.
}

\section{Gordon Blunn}

School of Pharmacy and Biomedical Sciences, University of Portsmouth, St Michael's Building, White Swan Road, Portsmouth, PO1 2DT, UK.

Jay Meswania

Fitzpatrick Referrals, Eashing, Godalming, Surrey, United Kingdom. GU7 2QQ.

Anita Sanghani-Kerai (corresponding author)

Fitzpatrick Referrals, Eashing, Godalming, Surrey, United Kingdom. GU7 2QQ.

Acknowledgements: The authors would like to thank the team at Fitzbionics for their help in developing the implant, and under the guidance of authors, medical writing support was provided by Dr Sarah Morgan.

Conflict of Interest: Noel Fitzpatrick is the inventor of the implant system and director of Fitzpatrick Referrals. However, this implant was not produced for commercial purposes so there was no financial gain from it. The rest of the authors declare no conflict of interest. 


\section{SUMMARY}

Aim: To describe the treatment of an infected segmental bone defect in a cat using a novel, custom-designed titanium implant seeded with adipose-derived stem cells (AdMSCs) to facilitate osseous ingrowth and preserve limb function.

Case report: Large bone defects occur secondary to trauma, infection, or neoplasia, and often result in amputation. We established a novel autologous AdMSC-impregnated trabecular metal spacer made using 3D printing, to bridge the distal tibia and metatarsal bones in the left pelvic limb of a cat that had previously undergone right pelvic limb amputation. 6months postoperatively, there was radiographic evidence of bone growth and implant integration.

Conclusion: A titanium spacer seeded with AdMSCs successfully encouraged bone ingrowth in a large defect site and successfully preserved limb function. However, further studies are needed to justify the use of differentiated stem cell impregnated mesh as a framework to bridge large bone defects.

\section{Key Words:}

Titanium, Bone Regeneration, Osseointegration, Adipose-Derived Mesenchymal Stem Cells

\section{INTRODUCTION}

Reconstruction of large segmental bone defects associated with significant trauma, neoplasia or infection are problematic and discontinuities in the bone are usually dealt with by amputation. The preferred treatment is an autologous bone graft, but there can be a limited supply of suitable bone, collection is also painful with risk of infection, haemorrhage, nerve damage and loss of function (Cipitria et al., 2013; Fröhlich et al., 2008). Scaffolds made from synthetic or natural biomaterials can overcome these problems, however in order to bridge large defects, they either need to be structurally competent or to be protected from functional loading by internal or external fixation. Additionally, to encourage the osteoinductive properties of these scaffolds, they can be seeded with stem cells (Berner et al., 2015; Cipitria et al., 2013; Fröhlich et al., 2008; M. Marcacci et al., 2007; Petite et al., 2000; Reichert et al., 2011) although a reliable solution has yet to be found for this technique.

Although titanium implants can be osseointegrated by promoting osteoblast proliferation and bone formation, they are neither osteoinductive nor osteoconductive, which are important properties if large defects are to be bridged by bone. The porosity of titanium implants can be manipulated to reduce stress shielding and to a certain extent this can encourage bone formation (Cheong, Fromme, Mumith, Coathup, \& Blunn, 2018; Zhang et al., 2018). Pobloth et al. successfully demonstrated that the mechanobiological optimisation of a 3D-manufactured titanium scaffold enhanced bone regeneration in large defects in an ovine model. This study reiterated the importance of lower stress shielding which led to earlier bridging of the defect (Pobloth et al., 2018). 
Mesenchymal stem cells are multipotent, can self-renew and, in the presence of an appropriate biologic stimulus, can differentiate into multiple tissues such as bone, cartilage and fat. In cats, these cells can be obtained from a number of different tissues such as bone marrow or adipose tissue (Lee et al., 2018; Panasophonkul, Samart, Kongon, \& Sathanawongs, 2017; Webb, Quimby, \& Dow, 2012). Human clinical studies have demonstrated the safety and efficacy of culture-expanded stem cells to enhance bone repair when seeded onto ceramic scaffolds (Maurilio Marcacci et al., 2007; Quarto et al., 2001).

The objective of this case report is to describe the use of a novel, custom endoprosthesis to facilitate osseous ingrowth into the implant in order to preserve limb function by replacing a bone defect following excision of a failed and infected reconstruction using bone graft and a plate in a cat.

\section{CASE REPORT}

An 8-year-old male neutered Maine Coon cat, presented with left tarsal swelling and a sudden reluctance to ambulate. The patient's right pelvic limb was amputated four years prior following a traumatic incident. Radiographs identified a chronic cranially dislocated left distal tibia with associated tibiotarsal joint collapse, remodelling and significant osseous proliferation. Radiolucencies were also evident throughout the tarsal bones and the distal tibia. These large lytic lesions were visible grossly on all tarsal joint bones upon surgical exploration. Histological evaluation of the lesions described a fibrotic, well-differentiated synovial hyperplasia with low-grade chronic inflammation. Antibiotic and analgesic therapies were provided and the patient was referred to a specialty orthopedic veterinary hospital.

On presentation, the left tarsus demonstrated signs of infection and microbial culture sensitivity testing identified Staphylococcus pseudointermedius, Enterobacter and Bacillus. Radiographs (Eklin Medical Systems, Santa Clara, California) and computed tomography (CT) scan (Toshiba Aquilion PRIME, 160 Slice, Toshiba Medical Systems, Tochigi, Japan) of the left pelvic limb confirmed the findings from the original referring vet. It was thought that this injury may have been triggered by previous trauma, resulting in degeneration, inflammation and infection. Radiographs also identified mild arthrosis of the left coxofemoral joint and evidence of osteoarthritis in both elbows.

The infection was treated orally every 12 hours with cephalexin $(22 \mathrm{mg} / \mathrm{kg})$ (Therios ${ }^{\circledR}$, Ceva Animal Health) on admission. Pradofloxacin $\left(3.37 \mathrm{mg} / \mathrm{kg}\right.$ ) (Veraflox ${ }^{\circledR}$, Bayer Animal Health GmbH, Leverkusen, Germany) was added every 24 hours upon receipt of the bacterial sensitivity profile. Although the swelling had improved and the draining tract had resolved after completion of a three week course of both antibiotics, as the cat remained unable to ambulate comfortably on his remaining pelvic limb, surgical arthrodesis was performed. All articular surfaces of the deformed tarsal bones were debrided. The talus was attached to the calcaneus using a $2 \mathrm{~mm}$ screw and a $2.0 \mathrm{~mm}$ medial plate was used to attach the talo-calcaneus to the distal row of tarsal and metatarsal bones. A 2.0/2.0 pantarsal arthrodesis plate was applied dorsally between the distal tibia and the third metatarsal bone to achieve alignment. Autogenous bone graft 
harvested from the remaining proximal aspect of the right femur was packed into the left tarsal arthrodesis site. A circular external skeletal fixator (IMEX ${ }^{\circledR}$ Veterinary, Inc, Texas, USA) was placed for additional stability.

Given the increased infection risk, daily therapy of pradofloxacin $(3.37 \mathrm{mg} / \mathrm{kg})$ was continued postoperatively. Despite the intraoperative microbial culture sensitivity testing result being negative, progressive surgical site swelling and development of purulent discharge was consistent with a recurrent infection. One week after surgery, the culture was repeated and tested positive for Enterococcus faecium. The surgical site was opened, flushed, and packed with Manuka honey (Manuka G, Jorgen Kruuse, Denmark). Once daily bandage changes and intravenous gentamicin $(8 \mathrm{mg} / \mathrm{kg})$ (Cidomycin, Sanofi, Berkshire, UK) was administered every 24 hours, in addition to ongoing pradofloxacin antimicrobial therapy and appropriate analgesia. After 5 days, the devascularized talus and distal row of tarsal bones were surgically removed along with a portion of the calcaneus, all of the bone graft, and the medial plate. The large defect was supported by the external frame and held in alignment by the dorsal bone plate [Figure 1A]. The open infection was treated with topical 1\% w/w chloramphenicol (Martindale Pharmaceuticals Ltd, Romford, UK) and continuous bandage changes with appropriate dressings. Antimicrobial therapies were replaced with twice daily $50 \mathrm{mg}$ oral minocycline ( $7 \mathrm{mg} / \mathrm{kg})(\mathrm{Actavis}$ Ltd, Barnstaple, UK) as dictated by the repeated culture sensitivity profile. An extended sensitivity profile returned 2 days later and the oral minocycline was replaced with daily intramuscular injections of oxytetracycline $(10 \mathrm{mg} / \mathrm{kg})$ (Engemycin 10\%, Intervet UK Ltd, Buckinghamshire, UK).

One week later, further surgical debridement was conducted and radio-opaque absorbable calcium sulfate pellets (Osteoset ${ }^{\mathrm{TM}}$ Resorbable mini-bead kit, Wright Medical group, Tennessee, USA) impregnated with vancomycin (Wockhardt, UK) and gentamicin (Cidomycin, Sanofi, Berkshire, UK) were inserted into the defect [Figure 1B]. The patient received daily bandage changes, intramuscular injection of oxytetracycline $\left(10 \mathrm{mg} / \mathrm{kg}\right.$ Engemycin ${ }^{\circledR} 10 \%$, Intervet, $\mathrm{UK})$ and oral administration of meloxicam $(0.05 \mathrm{mg} / \mathrm{kg})$ (Metacam, Boehringer Ingelheim, Ingelheim/Rhein, Germany), and an injectable analgesic when deemed medically appropriate for three weeks. As limb amputation was not an option in this case, a novel custom designed implant was considered the only remaining viable therapeutic approach. Owners were fully informed of this situation and gave full consent to the procedure.

Implant manufacture: A custom titanium pantarsal arthrodesis plate and curved titanium spacer were designed and manufactured by Fitzbionics Ltd (Surrey, UK), in accordance to a previous case series of pentatarsal arthrodesis on feline patients (Fitzpatrick, Sajik, \& Farrell, 2013). The design was developed from CT images using a 3-dimensional model in computer-aided design software (Mimics; Materialise, Sheffield, UK). The plate was manufactured by direct metal laser sintering of titanium alloy and was heat treated to relieve stresses. The plate was pre-contoured to a standing angle of $120^{\circ}$, with the largest cross sectional area at the bend, maximising plate strength at this level. Additionally, to minimise 
the risk of metatarsal bone stress fracture, the length of the plate is designed to occupy at least $70 \%$ of the metatarsal length, creating a long lever arm and transferring stress to the distal extremity of the metatarsal bone.

The spacer had a pore size of $600 \mu \mathrm{m}$ and a thickened mid-portion and a tapered proximal and distal end for force transfer [Figure 2C \& Figure 3].

Anaesthesia protocol: The patient was pre-medicated with intramuscular $2 \%$ acepromazine $(0.02 \mathrm{mg} / \mathrm{kg} \mathrm{IV})$ (Novartis, Surrey, UK) and methadone (0.2mg/kg IV) (Comfortan ${ }^{\circledR}$, Dechra, Skipton, UK), anaesthesia was induced with intravenous propofol (Abbott Animal Health, UK), given to effect, to allow a cuffed endotracheal tube to be placed and anaesthesia was maintained with Isoflurane (Abbott Animal Health, UK) in oxygen. Perioperative analgesia was achieved via the use of intermittent, intravenous bolus of methadone (Dechra, Skipton, UK).

Tissue harvest and Stem cell culture (AdMSCs): With informed consent from the patient's guardians, approximately $26 \mathrm{~g}$ of adipose tissue was harvested from the ventral abdominal region abaxial from midline. The adipose tissue was digested using $0.1 \%$ Collagenase (Sigma Aldrich, UK) at $37^{\circ} \mathrm{C}$ for 1 hour. After centrifugation at $2000 \mathrm{rpm}$ for 5 minutes, the pelleted cells were cultured in Dulbecco's Modified Eagle Media (DMEM) (Sigma Aldrich, UK) with 20\% Fetal Calf Serum (First Link Ltd, UK) and 1\% P/S until 80-90\% confluent, after which they were passaged.

After 2 weeks, 5 million passage 1 AdMSCs were seeded onto the titanium spacer. The titanium spacer was sterilized and placed in a 6 well plate. Cells suspended in growth media were evenly seeded dropwise throughout the porous structure of the implant. After 2 hours, the seeded implant was flooded in growth media and this was maintained for 2 days. In order to differentiate the AdMSCs along the osteoblastic lineage the media was supplemented with dexamethasone (Sigma Aldrich, UK), beta-glycerolphosphate (Sigma Aldrich, UK) and ascorbic acid (Sigma Aldrich, UK) for a further 3 days before implantation into the patient.

Surgical technique: Three weeks preceding implantation of the custom pantarsal arthrodesis device with a segmental spacer seeded with autologous cells, clinical signs consistent of an infection had abated. Antimicrobial therapies provided during that time consisted of intramuscular oxytetracycline $(10 \mathrm{mg} / \mathrm{kg})$ injections and local dissemination of gentamicin and vancomycin impregnated pellets. Prior to implantation, these partially absorbed pellets were flushed out of the defect. The plate was secured dorsally to the distal tibial and third metatarsal [Figure 2A], carefully avoiding the dorsal metatarsal artery. The spacer was positioned between the distal tibia and the proximal end of the residual metatarsus, prior to being fixed to the plate using screws [Figure 2B].

Postoperative management: Postoperative imaging revealed satisfactory alignment and stability [Figure 1C]. The patient remained hospitalised for 10 days after the operation. Although intra-operative culture was negative, and there was no evidence of infection, the preoperative antimicrobial regime was continued as a precaution. The patient was discharged with a 2-week course of twice daily oral minocycline $(7 \mathrm{mg} / \mathrm{kg})$ to replace the in-hospital ongoing 
intramuscular oxytetracycline injections. A 4 week course of once daily meloxicam $(0.05 \mathrm{mg} / \mathrm{kg})$ was also provided as the sole analgesic indicated for the patient's comfort.

Clinical follow-up: Two weeks postoperatively, the patient returned for examinatiosn. At which time the patient was using the limb comfortably despite an expected mechanical lameness. No discharge, erythema, swelling, or other evidence of infection was apparent. Skin apposition and healing was satisfactory and the skin sutures were removed. Surgical site was damaged 72 hours after removal of the sutures because of self-trauma. Although an Elizabethan collar was immediately replaced, the skin had already been damaged, resulting in a scab formation over the proximal aspect of the implant. One week after skin traumatization, four weeks postoperatively, the scab had fallen off and the metal implant was exposed, therefore requiring debridement, wound flushing and re-suturing of the skin. The patient was discharged with a repeated two-week course of twice-daily oral minocycline $(7 \mathrm{mg} / \mathrm{kg})$.

Two months after the custom surgery, the patient was using the limb well with no evidence of recurring infection. The patient was not on any medication. Sutures from the second incision closure were removed and repeat radiographs and $\mathrm{CT}$ scans were performed. The images indicated bone ingrowth between implant, tibia and tarsal bones consistent with bone bridging [Figure 1D]. Given these findings, activity restrictions were lifted. Six months post-implant surgery, the patient returned to full activity levels, was using the limb well and no longer received any medication. Radiographs showed continuous ossification between bone elements and the implant [Figure 1E], and CT scans showed excellent mesh integration with a large volume of remodeled bone spanning throughout the joint [Figure 1F, Figure 4]. The patient used the limb without identifiable pain although mechanical lameness was present. No additional complications were experienced. The patient died 18 months following surgery due to an unrelated illness. No necropsy was conducted because the patient was a family pet and died of natural causes, unrelated to the implant. The guardians elected for burial without any opportunity of histomorphometry of the implant.

\section{DISCUSSION}

Large bone defects are difficult to manage and can result in non-unions (Gomez-Barrena et al., 2015; Mehmood, Deshpande, Khan, Singh, \& Patil, 2017). In this case, the bone defect resulted from the removal of abnormal and infected bone, that occurred after trauma. To achieve successful healing of a large defect it is necessary to provide a suitable osteoinductive scaffold. Grafting materials such as hydroxyapatite are osteoconductive and can accelerate healing but they are not osteoinductive and alone would not be effective in bridging large bone defects. Consequently, it is important to ensure that the appropriate osteoinductive agents are integrated with these materials to encourage bone production at the defect site (Emara, Diab, \& Emara, 2015).

Ideal properties of graft material includes; osteoinduction induced by bone growth factors, osteogenesis encouraged by osteoprogenitor cells and osteoconduction provided by scaffolds (Dimitriou, Jones, McGonagle, \& 
Giannoudis, 2011; Virk \& Lieberman, 2012). Mechanical stability of the implant is crucial in order to provide stresses that favour bone formation (Giannoudis, Panteli, \& Calori, 2014; Pobloth et al., 2018). At the same time, the implant has to be strong enough to withstand the imposed loads. In this case, the preservation of left pelvic limb function was essential, as the patient had previously undergone amputation of the contralateral limb. This presented additional challenges, as there would be a greater load during healing, putting more stress on the repair, and increasing the risk of implant failure. The custom designed titanium spacer was incorporated with $600 \mu \mathrm{m}$ pores to encourage bone ingrowth (Pobloth et al., 2018; Taniguchi et al., 2016), aiding implant integration. This was further encouraged by the AdMSCs seeded on the implant, that were induced to form osteoblasts prior to implantation, which enhanced osteoconductive and osseoinductive properties, resulting in early osseointegration. Bone within the scaffold would remodel and be maintained by loading that is shared with the porous scaffold. Porous titanium implants have been investigated to enhance osseointegration in ovine diaphyseal replacement. Mummith and co-workers, 2017 demonstrated that selective laser sintered (SLS) porous collars increase the surface area for bone attachment allowing the direct ingrowth of more bone compared to solid grooved collars (Mumith et al., 2017). Even though the lifespan of this novel implant could only be assessed for 18 months, there was successful bone ingrowth in the implant.

Despite these available methods the concern for structural stability remains. Bone plates are often used to counteract the most structurally significant forces impacting the region. However, bone plates are incapable of completely correcting the forces acting along the central weight bearing axis of the bone without directly impeding bone growth. Additional potential complications included recurrence of infection, non-union, intrinsic implant design failure and compartment syndrome. This implant presented with technical difficulty during placement. Extra care was taken to ensure the dorsal metatarsal artery remained intact, as any damage to this vessel could result in avascular necrosis of the distal limb, effectively negating all repair efforts.

Titanium spacer seeded with AdMSCs differentiated in vitro along the osteoblastic lineage can encourage bone growth in large defect sites. However, the technology used in this single case is encouraging, it is expensive and technically difficult, therefore, additional research into its long-term outcomes and potential complications would be beneficial to support its future use and to support the use of differentiated stem cell impregnated mesh as a framework for repairing bone defects

Acknowledgements: The authors would like to thank the team at Fitzbionics for their help in developing the implant, and under the guidance of authors, medical writing support was provided by Dr Sarah Morgan. 
Conflict of Interest: Noel Fitzpatrick is the inventor of the implant system and director of Fitzpatrick Referrals. However, this implant was not produced for commercial purposes so there was no financial gain from it. The rest of the authors declare no conflict of interest.

Ethical approval: This work involved the use of non-experimental animals only (owned), and followed internationally recognised high standards ('best practice') of individual veterinary clinical patient care. Ethical Approval from a committee was not obtained, however written informed consent for the surgical and recovery procedure was obtained from the owners.

\section{REFERENCES}

Berner, A., Henkel, J., Woodruff, M. A., Steck, R., Nerlich, M., Schuetz, M. A., \& Hutmacher, D. W. (2015). Delayed minimally invasive injection of allogenic bone marrow stromal cell sheets regenerates large bone defects in an ovine preclinical animal model. Stem cells translational medicine, 4(5), 503-512.

Cheong, V. S., Fromme, P., Mumith, A., Coathup, M. J., \& Blunn, G. W. (2018). Novel adaptive finite element algorithms to predict bone ingrowth in additive manufactured porous implants. Journal of the Mechanical Behavior of Biomedical Materials, 87, 230-239. doi:https://doi.org/10.1016/i.jmbbm.2018.07.019

Cipitria, A., Reichert, J. C., Epari, D. R., Saifzadeh, S., Berner, A., Schell, H., . . . Hutmacher, D. W. (2013). Polycaprolactone scaffold and reduced rhBMP-7 dose for the regeneration of critical-sized defects in sheep tibiae. Biomaterials, 34(38), 9960-9968.

Dimitriou, R., Jones, E., McGonagle, D., \& Giannoudis, P. V. (2011). Bone regeneration: current concepts and future directions. BMC medicine, 9(1), 66. doi:10.1186/1741-7015-9-66

Emara, K. M., Diab, R. A., \& Emara, A. K. (2015). Recent biological trends in management of fracture non-union. World journal of orthopedics, 6(8), 623628. doi:10.5312/wjo.v6.i8.623

Fitzpatrick, N., Sajik, D., \& Farrell, M. (2013). Feline pantarsal arthrodesis using precontoured dorsal plates applied according to the principles of percutaneous plate arthrodesis. Veterinary and Comparative Orthopaedics and Traumatology, 26(05), 399-407.

Fröhlich, M., Grayson, W. L., Wan, L. Q., Marolt, D., Drobnic, M., \& Vunjak-Novakovic, G. (2008). Tissue engineered bone grafts: biological requirements, tissue culture and clinical relevance. Current stem cell research \& therapy, 3(4), 254264.

Giannoudis, P., Panteli, M., \& Calori, G. (2014). Bone Healing: The Diamond Concept. In G. Bentley (Ed.), European Instructional Lectures (Vol. 14, pp. 3-16): Springer Berlin Heidelberg.

Gomez-Barrena, E., Rosset, P., Lozano, D., Stanovici, J., Ermthaller, C., \& Gerbhard, F. (2015). Bone fracture healing: cell therapy in delayed unions and nonunions. Bone, 70, 93-101. doi:10.1016/j.bone.2014.07.033 
Lee, B. Y., Li, Q., Song, W. J., Chae, H. K., Kweon, K., Ahn, J. O., \& Youn, H. Y. (2018). Altered properties of feline adipose-derived mesenchymal stem cells during continuous in vitro cultivation. J Vet Med Sci, 80(6), 930-938. doi:10.1292/jvms.17-0563

Marcacci, M., Kon, E., Moukhachev, V., Lavroukov, A., Kutepov, S., Quarto, R., ... Cancedda, R. (2007). Stem cells associated with macroporous bioceramics for long bone repair: 6- to 7-year outcome of a pilot clinical study. Tissue Eng, 13(5), 947-955. doi:10.1089/ten.2006.0271

Marcacci, M., Kon, E., Moukhachev, V., Lavroukov, A., Kutepov, S., Quarto, R., ... Cancedda, R. (2007). Stem cells associated with macroporous bioceramics for long bone repair: 6-to 7-year outcome of a pilot clinical study. Tissue engineering, 13(5), 947-955.

Mehmood, M., Deshpande, S., Khan, S., Singh, P., \& Patil, B. (2017). Epidemiology of Delayed Union of Long Bones. J Trauma Treat, 6(370), 2167-1222.1000370.

Mumith, A., Coathup, M., Chimutengwende-Gordon, M., Aston, W., Briggs, T., \& Blunn, G. (2017). Augmenting the osseointegration of endoprostheses using lasersintered porous collars: an in vivo study. Bone Joint J, 99-b(2), 276-282. doi:10.1302/0301-620x.99b2.bjj-2016-0584.r1

Panasophonkul, S., Samart, P., Kongon, K., \& Sathanawongs, A. (2017). Phenotypic characteristics of feline adipose-derived stem cells affected by cell passage number. Pol J Vet Sci, 20(4), 651-660. doi:10.1515/pjvs-2017-0082

Petite, H., Viateau, V., Bensaid, W., Meunier, A., de Pollak, C., Bourguignon, M., ... Guillemin, G. (2000). Tissue-engineered bone regeneration. Nature biotechnology, 18(9), 959.

Pobloth, A.-M., Checa, S., Razi, H., Petersen, A., Weaver, J. C., Schmidt-Bleek, K., ... Schaser, K.-D. (2018). Mechanobiologically optimized 3D titanium-mesh scaffolds enhance bone regeneration in critical segmental defects in sheep. Science translational medicine, 10 (423), eaam8828.

Quarto, R., Mastrogiacomo, M., Cancedda, R., Kutepov, S. M., Mukhachev, V., Lavroukov, A., ... Marcacci, M. (2001). Repair of large bone defects with the use of autologous bone marrow stromal cells. $N$ Engl J Med, 344. doi:10.1056/nejm200102013440516

Reichert, J. C., Wullschleger, M. E., Cipitria, A., Lienau, J., Cheng, T. K., Schütz, M. A., ... Hutmacher, D. W. (2011). Custom-made composite scaffolds for segmental defect repair in long bones. International Orthopaedics, 35(8), 1229-1236.

Taniguchi, N., Fujibayashi, S., Takemoto, M., Sasaki, K., Otsuki, B., Nakamura, T., ... Matsuda, S. (2016). Effect of pore size on bone ingrowth into porous titanium implants fabricated by additive manufacturing: An in vivo experiment. Materials Science and Engineering: C, 59, 690-701. doi:https://doi.org/10.1016/i.msec.2015.10.069

Virk, M. S., \& Lieberman, J. R. (2012). Biologic adjuvants for fracture healing. Arthritis research \& therapy, 14(6), 225. doi:10.1186/ar4053

Webb, T. L., Quimby, J. M., \& Dow, S. W. (2012). In vitro comparison of feline bone marrow-derived and adipose tissue-derived mesenchymal stem cells. Journal of feline medicine and surgery, 14(2), 165-168. 


\section{Zhang, Y., Zhang, L., Sun, R., Jia, Y., Chen, X., Liu, Y., ... Feng, L. (2018). A new 3D printed titanium metal trabecular bone reconstruction system for early osteonecrosis of the femoral head. Medicine, 97(26).}

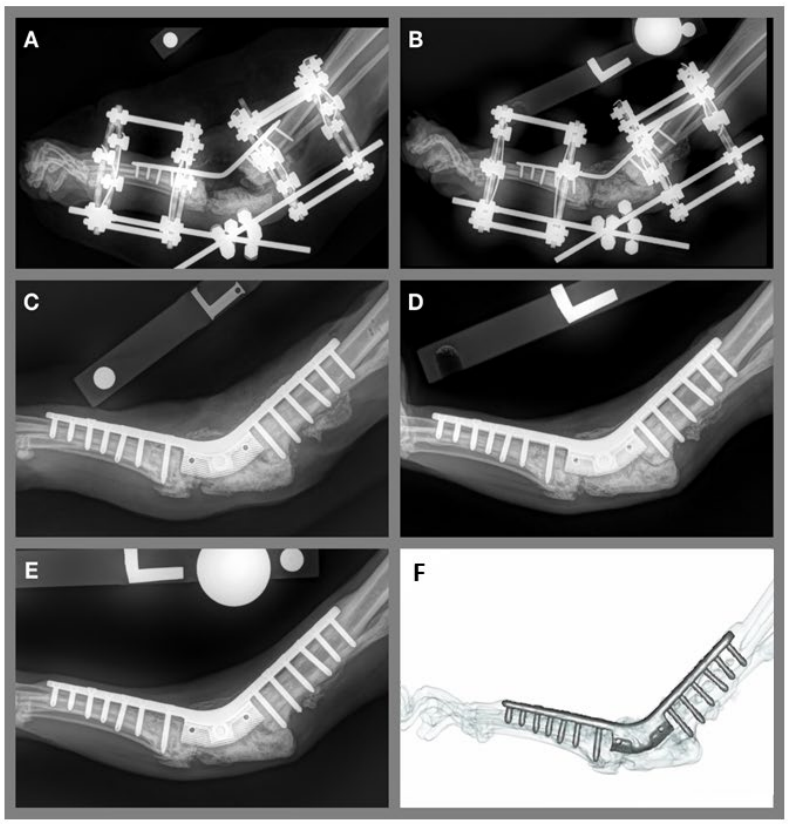

Figure 1: Radiograph immediately following surgical excision of devascularized tarsal bones and dorsal plate (A). Radiograph of the defect and enclosed radio-opaque calcium sulfate pellets taken prior to surgical implantation of the custom plate and spacer (B). Radiographs taken postoperatively (C), after 2 Months (D), and after 6 months (E) showing progressive osseous integration onto the custom implant and spacer. This is further displayed in the CT 3 dimensional reconstruction image $(\mathrm{F})$. 

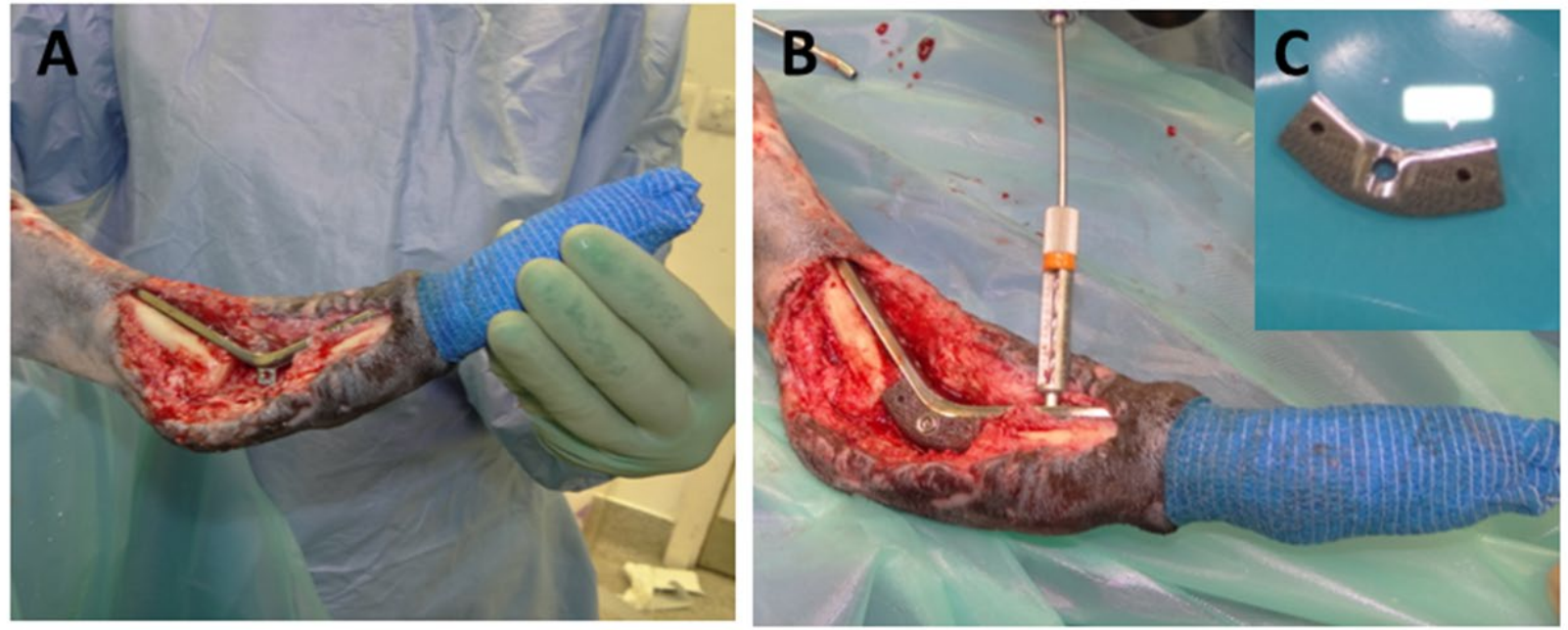

Figure 2: Intra-operative picture taken after plate placement (A), and after spacer fixation (B). The trabecular titanium spacer with seeded autologous stem cells is shown just prior to implantation. (C).
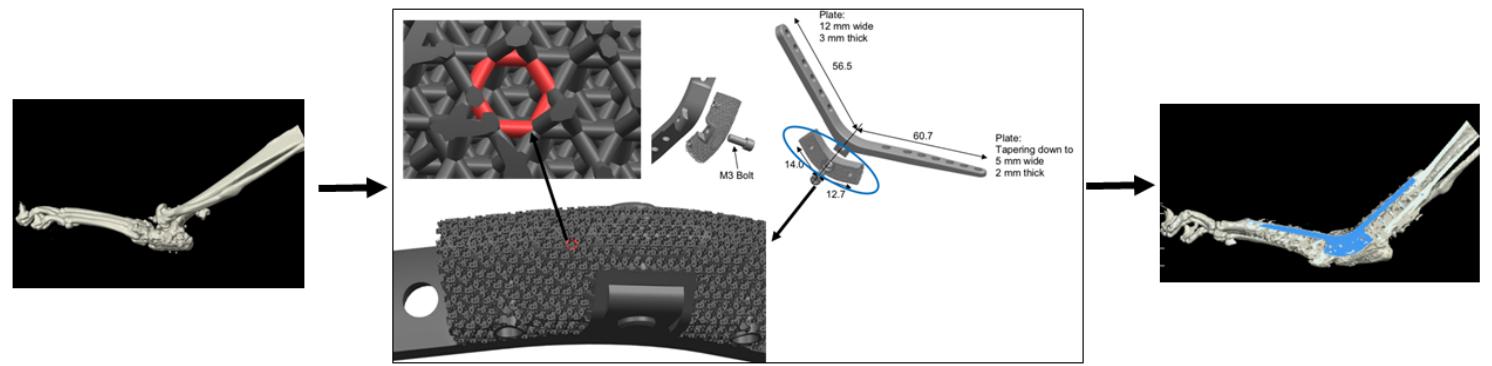

Figure 3: A step-by-step image of the implant development from the CT image acquisition, 3D rendering and the final result of the implant in the defect site. The porous membrane of the implant was made-up of hexagonal three dimensional matrix with pour size of $0.6 \mathrm{~mm}$ (shown in red) using Direct Metal Laser Sintering process. 


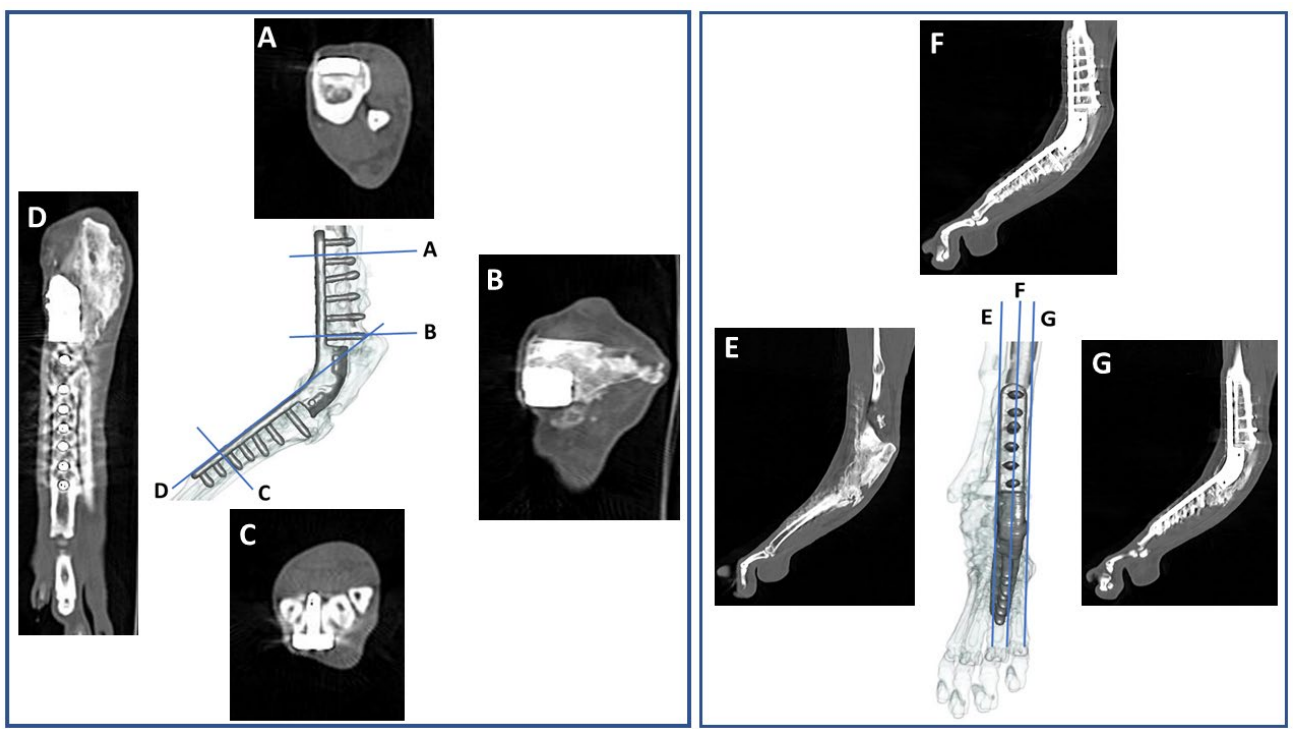

Figure 4: CT scan of the lateral sagittal (left) and dorsoplantar (right) showing bone ingrowth in different sections (from A-G) around the custom titanium plate and curved spacer after 6 months. 